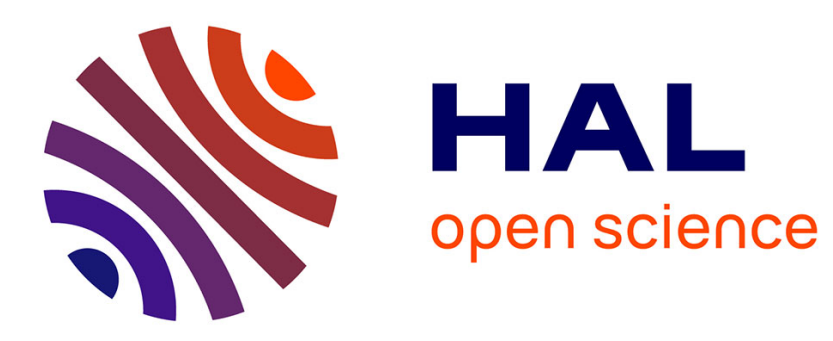

\title{
Influence of bending resistance on the dynamics of a spherical capsule in shear flow
}

Claire Dupont, Anne-Virginie Salsac, Dominique Barthes-Biesel, Marina

Vidrascu, Patrick Le Tallec

\section{- To cite this version:}

Claire Dupont, Anne-Virginie Salsac, Dominique Barthes-Biesel, Marina Vidrascu, Patrick Le Tallec. Influence of bending resistance on the dynamics of a spherical capsule in shear flow. Physics of Fluids, 2015, 27, pp.17. 10.1063/1.4921247 . hal-01160157

\section{HAL Id: hal-01160157 \\ https://hal.inria.fr/hal-01160157}

Submitted on 4 Jun 2015

HAL is a multi-disciplinary open access archive for the deposit and dissemination of scientific research documents, whether they are published or not. The documents may come from teaching and research institutions in France or abroad, or from public or private research centers.
L'archive ouverte pluridisciplinaire HAL, est destinée au dépôt et à la diffusion de documents scientifiques de niveau recherche, publiés ou non, émanant des établissements d'enseignement et de recherche français ou étrangers, des laboratoires publics ou privés. 
Influence of bending resistance on the dynamics of a spherical capsule in shear flow

C. Dupont, ${ }^{1,2}$ A.-V. Salsac, ${ }^{2, \text { a) }}$ D. Barthès-Biesel, ${ }^{2}$ M. Vidrascu, ${ }^{3,4}$ and P. Le Tallec ${ }^{1}$

1) Solid Mechanics Laboratory (UMR CNRS 7649), Ecole Polytechnique, 91128 Palaiseau Cedex, France

${ }^{2)}$ Biomechanics and Bioengineering Laboratory (UMR CNRS 7338),

Université de technologie de Compiègne - CNRS, Sorbonne universités, CS 60319, 60203 Compiègne, France

3) REO project-team, Inria-Rocquencourt, B.P. 105, 78153 Le Chesnay cedex, France

4) REO project-team (LJLL, UMR CNRS 7958), UPMC Université de Paris VI, 75005 Paris, France 
The objective of the paper is to study the effect of wall bending resistance on the motion of an initially spherical capsule freely suspended in shear flow. We consider a capsule with a given thickness made of a three-dimensional homogeneous elastic material. A numerical method is used to model the fluid-structure interactions coupling a boundary integral method for the fluids with a shell finite element method for the capsule envelope. For a given wall material, the capsule deformability strongly decreases when the wall bending resistance increases. But, if one expresses the same results as a function of the two-dimensional mechanical properties of the mid-surface, which is how the capsule wall is modeled in the thin-shell model, the capsule deformed shape is identical to the one predicted for a capsule devoid of bending resistance. The bending rigidity is found to have a negligible influence on the overall deformation of an initially spherical capsule, which therefore depends only on the elastic stretching of the mid-surface. Still, the bending resistance of the wall must be accounted for to model the buckling phenomenon, which is observed locally at low flow strength. We show that the wrinkle wavelength is only a function of the wall bending resistance and provide the correlation law. Such results can then be used to infer values of the bending modulus and wall thickness from experiments on spherical capsules in simple shear flow.

PACS numbers: 47.11.Hj, 87.10.Kn, 87.15.La, 87.85.G, 87.85.gf

a)Electronic mail: a.salsac@utc.fr; www.utc.fr/bmbi 


\section{INTRODUCTION}

Capsules, which consist of an internal liquid droplet enclosed by a membrane, have numerous applications in bioengineering, cosmetics and pharmaceutics: they are designed to protect fragile or volatile substances and to control their liberation in the external media. They are also used as biomimetic models of cells such as red blood cells. One classical technique to produce deformable capsules relies on interfacial polymerization of an emulsion. The fabricated capsules are typically quasi-spherical at rest. The membrane thickness and mechanical properties depend on the fabrication process ${ }^{1-4}$. For many processes such as interfacial cross-linking, the capsule wall thickness results to be small as compared to the radius. But determining its exact value remains technically challenging, as it tends to be sub-micronic.

The dynamics of an initially spherical capsule subjected to a shear flow are of practical interest and have thus been studied extensively over the past years (see the review ${ }^{5}$ ). It has been observed experimentally ${ }^{4,6-8}$ that at steady state, a spherical capsule is elongated in the straining direction by the hydrodynamic stresses exerted by the external flows, while the membrane rotates around the deformed shape because of the flow vorticity. However, for low flow strength, the capsule membrane is compressed in the equatorial region and thus becomes wrinkled ${ }^{4,8}$. Membrane wrinkling and buckling weaken the capsule membrane and may lead to fatigue breakup. It is thus important to predict these phenomena in order to avoid/provoke the membrane rupture depending on the application.

In order to understand the complex behavior of a spherical capsule in an external flow, numerical models of the fluid-structure interaction have been developed where the membrane is treated as a zero thickness elastic surface devoid of bending resistance ${ }^{5}$. The observed experimental phenomena of elongation along the viscous strain direction, membrane rotation and tendency towards buckling at low shear rates are recovered by the numerical models. The models show clearly how the capsule motion and deformation depend on the flow strength relative to the elastic forces and on the viscosity ratio between the external and internal fluids ${ }^{9-13}$.

In order to study the buckling and wrinkling phenomena in a rigorous way, the capsule wall bending stiffness must be accounted for in the model. Most numerical models that include bending effects have decomposed the wall strain energy into the sum of a membrane 
elastic energy and of a bending energy computed from the local curvature ${ }^{14,15}$. The bending modulus values, which have been used, are extremely high and have no relation with the bulk shear modulus of the wall. Such a decoupled energy-based model is likely to be relevant for objects like the red blood cells, which have a composite membrane consisting of a lipid bilayer lined by a protein network. Its relevance can, however, be questioned for artificial capsules with a thin homogeneous membrane, especially when irrealistic values of bending modulus are modeled.

Another approach has been to consider the membrane wall as a three-dimensional homogeneous material and to use the thin shell approximation. Le \& $\operatorname{Tan}^{16}$ have developed such a thin shell model, but they have restricted the analysis to a Kirchhoff-Love kinematical assumption (i.e. no transverse shear) and only considered one small value of wall thickness. The effect of the wall bending resistance on the dynamics of a capsule suspended in an external flow is thus still an open question.

The objective of the study is to analyze the deformation of an initially spherical capsule in a simple shear flow, assuming that the wall is thin and made of a three-dimensional homogeneous elastic material with uniform thickness that resists both membrane and bending deformations. The equations governing the problem are solved numerically coupling a boundary integral method to compute the flow of the internal and external liquids with a shell finite element method to compute the deformation of the capsule wall, as outlined in sections II and III. We show how, for a given wall material, the thickness influences the deformation under a given flow strength in section IV and analyze which physical phenomena govern the capsule global deformation. We finally discuss the formation of wrinkles on the membrane and show how their wavelength is related to the bending resistance and correlatively to the wall thickness.

\section{PROBLEM FORMULATION}

We consider an initially spherical capsule enclosed by a three-dimensional homogeneous incompressible wall of thickness $\alpha \ell(\alpha<1)$. The capsule radius $\ell$ is measured from the capsule mid-surface $\mathcal{S}_{t}$, which is located at the middle of the wall thickness. The wall is made of a material, which is supposed to be hyperelastic at moderate deformation with bulk shear modulus $G$ and Poisson ratio $\nu=1 / 2$. The capsule is suspended in a simple shear 
flow in the $x y$-plane with undisturbed velocity field

$$
\underline{v}^{\infty}=\dot{\gamma} y \underline{e}_{x}
$$

where $\dot{\gamma}$ is the shear rate. The inner and outer fluids have the same viscosity $\mu$ and density.

By convention, all the quantities are denoted by capital letters in the reference state and by lowercase letters in the deformed state. The surface tensor components are denoted with Greek indices and the 3D tensor components with Latin indices. We adopt the Einstein summation convention on repeated indices. The subscript $s$ is used to designate quantities calculated on the mid-surface $\mathcal{S}_{t}$.

\section{A. Wall mechanics}

Due to the small mass of the capsule wall, inertia effects are neglected when solving for the wall deformation. We assume the wall to be sufficiently thin to be modeled as a thin shell with mid-surface $\mathcal{S}_{t}$, as described, among others, by Chapelle \& Bathe ${ }^{17}$ and briefly reviewed thereafter. The principle of the thin shell consists of evaluating all the quantities of interest on the mid--surface.

The position of a material point in the shell is defined by the independent curvilinear coordinates $\left(\xi^{1}, \xi^{2}, \xi^{3}\right)$. In the reference non-deformed state (time $t=0$ ), the position of a point $\mathrm{M}$ of the mid-surface is given by

$$
\underline{O M}=\underline{\varphi}\left(\xi^{1}, \xi^{2}\right)
$$

with $O$ the chosen origin. It is convenient to introduce the local covariant base $\left(\underline{A}_{1}, \underline{A}_{2}, \underline{A}_{3}\right)$ defined as

$$
\underline{A}_{\alpha}=\frac{d \underline{\varphi}\left(\xi^{1}, \xi^{2}\right)}{d \xi^{\alpha}}=\underline{\varphi}, \alpha \quad \text { and } \quad \underline{A}_{3}=\frac{\underline{A}_{1} \times \underline{A}_{2}}{\left\|\underline{A}_{1} \times \underline{A}_{2}\right\|},
$$

where $\underline{A}_{3}$ is the unit normal vector. The contravariant base $\left(\underline{A}^{1}, \underline{A}^{2}, \underline{A}^{3}\right)$ is defined by $A^{\alpha} \cdot A_{\beta}=\delta_{\beta}^{\alpha}$ with $\delta_{\beta}^{\alpha}$ the Kronecker tensor and $\underline{A}^{3}=\underline{A}_{3}$. The same quantities are defined in the deformed state using lowercase letters: the local covariant and contravariant bases are respectively denoted $\left(\underline{a}_{1}, \underline{a}_{2}, \underline{a}_{3}\right)$ and $\left(\underline{a}^{1}, \underline{a}^{2}, \underline{a}^{3}\right)$. In the reference configuration, the threedimensional position $\underline{X}$ of a material point within the capsule wall is then

$$
\underline{X}\left(\xi^{1}, \xi^{2}, \xi^{3}\right)=\underline{\varphi}\left(\xi^{1}, \xi^{2}\right)+\xi^{3} \underline{A}_{3}
$$


where $\left|\xi^{3}\right| \leq \alpha \ell / 2$. In the deformed configuration, the new position $\underline{x}$ of a material point differs from the original position $\underline{X}$ through an unknown displacement field $\underline{u}$ :

$$
\underline{x}(\underline{X}, t)=\underline{X}+\underline{u}(\underline{X}, t) .
$$

The thin shell theory ${ }^{17}$ assumes that the displacement field satisfies the Reissner-Mindlin kinematic assumption, i.e. a material line initially orthogonal to the mid-surface remains straight and unstretched during deformation but does not remain orthogonal to the deformed mid-surface. All the terms depending on $\left(\xi^{3}\right)^{2}$ are thus neglected in the expression of the displacement $\underline{u}$, which can then be written as

$$
\underline{u}\left(\xi^{1}, \xi^{2}, \xi^{3}, t\right)=\underline{u}_{s}\left(\xi^{1}, \xi^{2}, t\right)+\xi^{3} \theta_{\lambda}\left(\xi^{1}, \xi^{2}, t\right) \underline{a}^{\lambda}\left(\xi^{1}, \xi^{2}, t\right) .
$$

The first term $u_{s}$ represents the displacement of the mid-surface at coordinates $\left(\xi^{1}, \xi^{2}\right)$. In the second term, the angles $\theta_{1}$ and $\theta_{2}$ are the rotation angles of the line passing by the point $\left(\xi^{1}, \xi^{2}\right)$ of the mid--surface and perpendicular to the latter around the tangential vectors $\underline{a}^{2}$ and $\underline{a}^{1}$, respectively. To simplify the notations in the following, we introduce the rotational surface vector $\underline{\theta}\left(\xi^{1}, \xi^{2}, t\right)=\theta_{\lambda}\left(\xi^{1}, \xi^{2}, t\right) \underline{a}^{\lambda}\left(\xi^{1}, \xi^{2}, t\right)$.

The deformation gradient $\underline{\underline{F}}=\frac{\partial \underline{x}}{\partial \underline{X}}$ and the Green-Lagrange strain tensor $\underline{\underline{e}}=\frac{1}{2}\left(\underline{\underline{F}}^{T}\right.$. $\underline{\underline{F}}-\mathbb{I}$ ) induced by this displacement field are then deduced from Eq. (5-6) (see Appendix A). Asymptotic analysis ${ }^{17}$ shows that the shell is undergoing plane stress at first order.

We assume that the wall material is hyperelastic: the second Piola-Kirchhoff stress tensor $\stackrel{\sum}{\equiv}$ inside the wall is a given function of the Green-Lagrange strain tensor through the strain energy density function. For a linear isotropic material, the stress-strain relationship reduces to the generalized Hooke's law

$$
\underline{\underline{\underline{N}}}=2 G\left(\underline{\underline{e}}+\frac{\nu}{1-\nu} \operatorname{tr} \underline{\underline{e}}\right) .
$$

The Cauchy stress tensor $\underline{\underline{\sigma}}$, which contains forces per unit area of deformed material, is related to the Piola-Kirchhoff stress tensor by

$$
\underline{\underline{\sigma}}=\frac{1}{\operatorname{det} \underline{\underline{F}}} \underline{\underline{F}} \cdot \underline{\underline{\Sigma}} \cdot \underline{\underline{F}}^{T}
$$

It follows that, even for a linear material, $\underline{\underline{\sigma}}$ is a non-linear function of strain. 
The wall equilibrium equations are then

$$
\left\{\begin{array}{l}
\nabla \cdot \underline{\underline{\sigma}}=\underline{0} \text { inside the wall, } \\
\underline{\underline{\sigma}} \cdot \underline{a}_{3}=\underline{q}^{+} \text {on the external wall surface } \mathcal{S}_{t}^{+} \\
\underline{\underline{\sigma}} \cdot \underline{a}_{3}=-\underline{q}^{-} \text {on the internal wall surface } \mathcal{S}_{t}^{-}
\end{array}\right.
$$

where $\underline{q}^{+}$(respectively $\underline{q}^{-}$) is the viscous load per unit deformed area exerted by the external (respectively internal) fluid flow. They can be rewritten using the principle of virtual work, which dictates that the work done by the external loads acting on a deformable body are equal to the virtual change in internal strain energy. Let $\mathcal{V}$ be the Sobolev space $H^{1}\left(\mathcal{S}_{t}, \mathbb{R}^{3}\right)$. For any virtual displacement $\underline{\hat{u}}_{s} \in H^{1}\left(\mathcal{S}_{t}, \mathbb{R}^{3}\right)$ satisfying the Reissner-Mindlin assumption (Eq. 6) and any virtual rotation $\underline{\hat{\theta}} \in H^{1}\left(\mathcal{S}_{t}, \mathbb{R}^{2}\right)$, we have from Eq. (9)

$$
\int_{\mathcal{S}_{t}^{+}} \underline{\hat{u}}\left(\underline{\hat{u}}_{s}, \underline{\hat{\theta}}\right) \cdot \underline{q}^{+} d S-\int_{\mathcal{S}_{t}^{-}} \underline{\hat{u}}\left(\underline{\hat{u}}_{s}, \underline{\hat{\theta}}\right) \cdot \underline{q}^{-} d S=\int_{V} \underline{\underline{\hat{e}}}\left(\underline{\hat{u}}_{s}, \underline{\hat{\theta}}\right): \underline{\underline{\sigma}} d V,
$$

where $V$ is the shell wall volume in the deformed state, $\underline{\hat{u}}$ an arbitrary kinematically admissible virtual displacement and $\underline{\underline{e}}$ the corresponding virtual strain tensor. On $\mathcal{S}_{t}^{+}$, the virtual displacement reads

$$
\underline{\hat{u}}\left(\xi^{1}, \xi^{2}, t\right)=\underline{\hat{u}}_{s}\left(\xi^{1}, \xi^{2}, t\right)+\frac{\alpha \ell}{2} \underline{\hat{\theta}}\left(\xi^{1}, \xi^{2}, t\right),
$$

$+\alpha \ell / 2$ being replaced by $-\alpha \ell / 2$ on $\mathcal{S}_{t}^{-}$.

Since the capsule wall is treated as a thin shell for both the real and virtual displacement fields, the virtual work of the external load can be expressed in terms of a load $\underline{q}$ evaluated on the mid-surface

$$
\int_{\mathcal{S}_{t}} \underline{\hat{u}}\left(\underline{\hat{u}}_{s}, \underline{\hat{\theta}}\right) \cdot \underline{q} d S=\int_{V} \underline{\underline{\hat{e}}}\left(\underline{\hat{u}}_{s}, \underline{\hat{\theta}}\right): \underline{\underline{\sigma}} d V
$$

where $\underline{q}$ is the jump of viscous traction forces exerted by the fluids. The difference between the membrane and shell models is that, in the membrane model, all the terms which are $O(\alpha)$ are ignored, so that the membrane displacement is only given by $\underline{u}_{s}\left(\xi^{1}, \xi^{2}, t\right)$. In the shell model, the rotational and three-dimensional effects across the wall thickness are taken into account in the displacement (Eq. 6). These effects are included in the right-hand side term of Eq. (12) and lead to a resistance to bending, which is quantified by a bending modulus $M_{b}$. For a homogeneous material that follows the generalized Hooke's law (Eq. 7), $M_{b}$ is given by

$$
M_{b}=\frac{G}{6(1-\nu)}(\alpha \ell)^{3}
$$


It follows that for the same deformation of the mid-surface, the expression of the load $\underline{q}$ is different for the membrane and shell models.

\section{B. Internal and external flows}

Owing to the small capsule size, the Reynolds number $R e=\rho \ell^{2} \dot{\gamma} / \mu$ is very small. The internal and external flows are thus governed by the Stokes equations. For thin walls, when the inner and outer fluids are of equal viscosity, the local velocity of the mid-surface points is then equal to the following integral of the viscous traction jump over the deformed capsule surface $\mathcal{S}_{t}$

$$
\forall \underline{x}_{s} \in \mathcal{S}_{t}, \quad \underline{v}\left(\underline{x}_{s}\right)=\underline{v}^{\infty}\left(\underline{x}_{s}\right)-\frac{1}{8 \pi \mu} \int_{S_{t}}\left(\frac{\mathbb{I}}{\|\underline{r}\|}+\frac{\underline{r} \otimes \underline{r}}{\|\underline{r}\|^{3}}\right) \cdot \underline{q}\left(\underline{y}_{s}\right) d S,
$$

where $\underline{v}^{\infty}$ is the undisturbed flow velocity. The vector $\underline{r}=\underline{x}_{s}-\underline{y}_{s}$ is the distance vector between the point $\underline{x}_{s}$, where the velocity vector is calculated, and the point of integration

$\underline{y}_{s}$ located on the surface $\mathcal{S}_{t}$. The wall and fluid mechanics are coupled through $\underline{q}$ and through the kinematic condition, which relates the wall velocity to the time derivative of the displacement field at the capsule mid-surface:

$$
\forall \underline{x}_{s} \in \mathcal{S}_{t}, \quad \underline{v}\left(\underline{x}_{s}\left(\xi^{1}, \xi^{2}, t\right)\right)=\frac{\partial}{\partial t} \underline{u}_{s}\left(\xi^{1}, \xi^{2}, t\right)
$$

Note that the velocities of the inner and outer fluids must be equal in order to keep the membrane shear finite.

\section{Problem parameters}

The capsule dynamics are governed by the relative wall thickness $\alpha$ and by the bulk capillary number

$$
C a_{v}=\mu \dot{\gamma} / G
$$

which compares the viscous to the elastic forces. When the wall is infinitely thin $(\alpha \ll 1)$, it is customary to introduce a surface shear modulus $G_{s}=G \alpha \ell$, to which corresponds a surface capillary number

$$
C a_{s}=\mu \dot{\gamma} \ell / G_{s}=C a_{v} / \alpha
$$


This surface capillary number is classically used to study the dynamics of capsules without bending resistance. When the bending resistance of the wall is accounted for, a bending number $K_{b}$ can be introduced to measure the relative importance of bending and shearing effects:

$$
K_{b}=\frac{M_{b}}{\alpha G \ell^{3}}=\frac{M_{b}}{G_{s} \ell^{2}}
$$

When we consider the capsule wall to be composed of a homogeneous incompressible material, the bending number is simply $K_{b}=\alpha^{2} / 3$ as predicted from Eq. (13).

\section{NUMERICAL METHOD}

To solve the fluid-structure interaction problem (Eq. 7, 8, 12, 14, 15), we iteratively couple $(i)$ a shell finite element method to solve the solid problem (i.e. Eq. 7, 8, 12) knowing the mid-surface displacement $\underline{u}_{s}$ and find the value of the viscous load $\underline{q}$ acting on the capsule wall, and (ii) a boundary integral method to compute the local velocity of the mid-surface points using Eq. (14) for a given deformed capsule geometry and the viscous load transferred by the solid solver. We then update the position $\underline{u}_{s}$ of the mid-surface by integrating in time Eq. (15) using a first-order explicit Euler scheme. The underlying philosophy of the method is similar to the one designed by Walter et al. ${ }^{12}$ to simulate the dynamics of a capsule devoid of bending resistance. But, when the wall is modeled as a thin shell with finite thickness and bending resistance, the three-dimensional displacement field $\underline{u}$ is no longer known at the beginning of each iteration (only $\underline{u}_{s}$ is): the rotation vector $\underline{\theta}$ needs to be solved for.

Since the evolution equation (Eq. 15) is integrated in time with an explicit scheme, the numerical method is stable only for sufficiently small time steps $\Delta t$. The stability criterion

$$
\dot{\gamma} \Delta t<\mathcal{O}\left(\frac{\Delta x C a_{s}}{\ell}\right)
$$

previously defined by Walter et $a .^{18}$ for a capsule without bending resistance remains valid, $\Delta x$ denoting the mesh size. The introduction of bending does not modify the stability analysis, since the problem is inertialess. 


\begin{tabular}{|c|cccccc|}
\hline$N_{E}$ & 320 & $\mathbf{5 1 2}$ & 1280 & $\mathbf{2 0 4 8}$ & 5120 & $\mathbf{8 1 9 2}$ \\
$N_{N}$ & 162 & $\mathbf{2 5 8}$ & 642 & $\mathbf{1 0 2 6}$ & 2562 & $\mathbf{4 0 9 8}$ \\
$\Delta x / \ell$ & 0.3 & $\mathbf{0 . 2 3}$ & 0.15 & $\mathbf{0 . 1 2}$ & 0.075 & $\mathbf{0 . 0 6}$ \\
\hline
\end{tabular}

TABLE I. Number of nodes $N_{N}$ and characteristic mesh size $\Delta x / \ell$ as a function of the number of elements $N_{E}$. The meshes obtained from an octahedron are written in boldface.

(a)

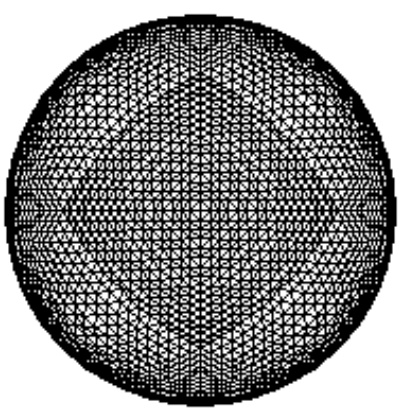

(b)

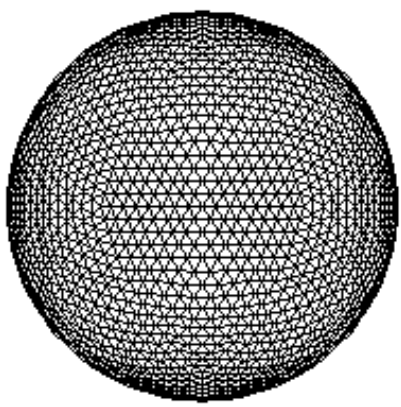

FIG. 1. Typical meshes, respectively with 8192 and 5120 elements, obtained from the initial inscription of $(a)$ an octahedron or of $(b)$ an icosahedron in a sphere.

\section{A. Discretization of the capsule wall}

At time $t=0$, the undeformed mid-surface of the capsule wall is discretized with linear triangular shell elements obtained by inscribing either an icosahedron (regular polyhedron with 20 triangular faces) or an octahedron (regular polyhedron with 8 triangular faces) in a sphere, and sequentially subdividing the triangular elements and projecting the resulting nodes on the spherical mid-surface. We denote $N_{E}$ and $N_{N}$ the number of elements and nodes respectively and $\Delta x / \ell$ the characteristic mesh size. The number of nodes and the average mesh size used in this study are given in Table I as a function of the number of elements. The mesh obtained from an octahedron is symmetrical about two meridians, while the mesh obtained from an icosahedron is more homogeneous (Figure 1).

\section{B. Shell finite element procedure}

The wall mid-surface equilibrium problem (Eq. 7, 8, 12) is treated with the shell finite element library Shelddon designed by Inria ${ }^{19}$. The shell finite element solver is coupled with 
the fluid solver ${ }^{12,13,18}$ by means of a Parallel Virtual Machine protocol.

The wall mid-surface is discretized with triangular shell elements of the MITC family (Mixed Interpolation Tensorial Components) with three nodes (one at each vertex), which are typically referred to as MITC3. Their formulation is based on standard linear shape functions $\lambda^{(p)}(p \in\{1,2,3\})$ interpolating both the mid-surface displacement $\underline{u}_{s}$ and the rotations $\theta_{\lambda}$. The unit normal vector $\underline{a}_{3}^{(p)}$ is calculated at each node $p$. The position of a point of the wall is determined by the local Cartesian coordinates $(r, s, z)$, where $(r, s)$ are the intrinsic coordinates in the plane element (defined such that $r, s$ and $1-r-s \in[0,1]$ ) and $z$ is the coordinate along $\underline{a}_{3}(z \in[-1,1])$. The position vector $\underline{x}$ inside an element thus reads

$$
\underline{x}(t)=\sum_{p=1}^{3} \lambda^{(p)}(r, s)\left(\underline{x}_{s}^{(p)}(t)+z \frac{\alpha \ell}{2} \underline{a}_{3}^{(p)}(t)\right),
$$

and the corresponding virtual displacement

$$
\underline{\hat{u}}=\sum_{p=1}^{3} \lambda^{(p)}(r, s)\left(\underline{\hat{u}}_{s}^{(p)}+z \frac{\alpha \ell}{2} \underline{\hat{\theta}}^{(p)}\right) .
$$

The MITC technique is based on separate interpolations of the in-plane and out-of-plane components (e.g. $e_{\alpha 3}$ components) of the strain tensor, which are then connected at specific tying points. The MITC shell finite elements are shown to behave properly both for bending and for membrane dominated problems and satisfy the following properties:

- The finite element discretization leads to no spurious zero energy mode when solving the problem.

- The finite element solutions converge to the solution of the mathematical model, when the mesh size $\Delta x / \ell$ tends to zero.

- The shell is free of shear and membrane locking.

- The solution accuracy is independent of the shell thickness parameter.

- The matrices do not depend on the element orientation.

Details on the MITC technique can be found in Lee \& Bathe $^{20}$ and on the discretization of the equations in Chapelle \& Bathe ${ }^{17}$. 
Let $v_{X_{j}}^{(p)}$ denote the value of the $j^{\text {th }}$ Cartesian component of any vector $\underline{v}$ at node $p$ and $\{v\}$ the array of size $3 N_{N}$ containing the Cartesian components of the vector nodal values. The wall equilibrium equation (Eq. 12) can be discretized element-wise as:

$$
\sum_{e l} \hat{u}_{X_{j}}^{(p)}\left(\int_{S_{e l}} \lambda^{(p)} \lambda^{(q)} d S\right) q_{X_{j}}^{(q)}=\sum_{e l} \int_{V_{e l}} \hat{e}_{\alpha \beta} \sigma^{\alpha \beta} d V .
$$

The discretization of the right-hand-side of Eq. (12) is more complex than in the case of the left-hand-side, since the Green-Lagrange strain tensor and the second Piola-Kirchhoff stress tensor have to be expressed according to the nodal displacements. More details can be found in Batoz \& Dhatt ${ }^{21}$ (p. $\left.324-325\right)$.

After assembling the arrays and matrices across the mesh elements, the discretized form of Eq. (12) becomes

$$
\{\hat{u}\}^{T}[M]\{q\}=\{\hat{u}\}^{T}\{R\}\left(\left\{u_{s}\right\},\{\theta\}\right),
$$

where $[M]$ is a matrix that has the same structure as a mass matrix and the vector $\{R\}$ contains the elastic coefficients of the wall material.

As the equation must be satisfied for any virtual displacement, it can be simplified as

$$
[M]\{q\}=\{R\}\left(\left\{u_{s}\right\},\{\theta\}\right)
$$

or more precisely, by separating the membrane degrees of freedom from the rotational degrees of freedom,

$$
\left\{\begin{array}{l}
\{R\}_{s}\left(\left\{u_{s}\right\},\{\theta\}\right)-[M]_{s}\{q\}=\{0\}_{s} \\
\{R\}_{\theta}\left(\left\{u_{s}\right\},\{\theta\}\right)=\{0\}_{\theta}
\end{array}\right.
$$

In this system, the mid-surface displacement degrees of freedom $\left\{u_{s}\right\}$ are known from the solution $\underline{x}_{s}$ of the fluid problem and the integration of Eq. (15), while the viscous loads $\{q\}$ and the rotations $\{\theta\}$ are unknown. To solve this system, we first solve the nonlinear equation $(24 b)$ in $\{\theta\}$ by a Newton's method and then obtain the load exerted by the fluids on the membrane by a direct inversion of Eq. (24a).

\section{Numerical accuracy and convergence}

To verify the temporal and spatial convergence of the coupling method, we consider the motion of an initially spherical capsule of thickness ratio $\alpha=0.01$, subjected to a simple 


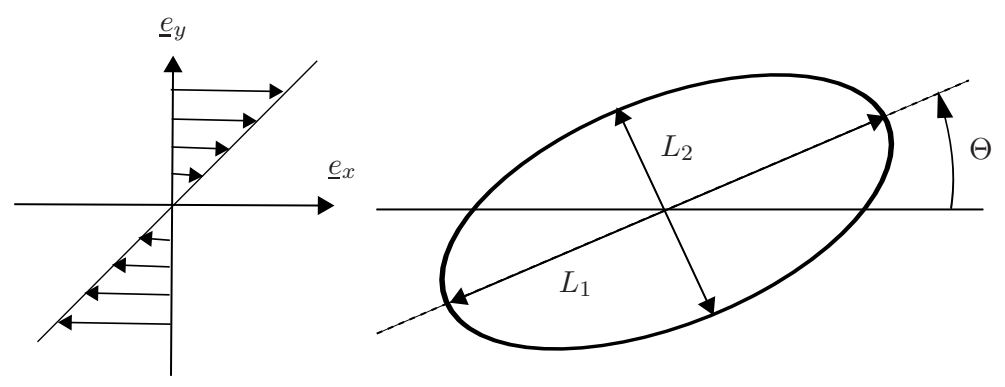

FIG. 2. Representation in the shear plane $\left(\underline{e}_{x}, \underline{e}_{y}\right)$ of the ellipsoid of inertia of the deformed midsurface of an initially spherical capsule. $L_{1}$ and $L_{2}$ are the lengths of the two principal axes in the shear plane. The angle $\Theta$ gives the inclination of the deformed capsule.

shear flow at $C a_{s}=0.6$. At steady state, the capsule mid--surface takes a quasi-ellipsoidal shape, which can be approximated by its ellipsoid of inertia. If we call $L_{1}$ and $L_{2}$ the lengths of the two principal axes of the ellipsoid of inertia in the shear plane (Figure 2), the capsule deformation at steady state can be measured by the Taylor parameter $D_{12}^{\infty}$

$$
D_{12}^{\infty}=\frac{L_{1}-L_{2}}{L_{1}+L_{2}}
$$

There is no analytical solution for the large deformation of a spherical capsule in a shear flow. To study the spatial convergence of the numerical method, we thus take as reference value $D_{12}^{\infty, r e f}$ the value of $D_{12}^{\infty}$ obtained with the smallest time step $\left(\dot{\gamma} \Delta t=1 \times 10^{-4}\right)$ and the most refined mesh size $\left(N_{E}=8192\right)$ at $\dot{\gamma} \Delta t=4$, at which the steady state is reached. We define the relative numerical error as

$$
\epsilon_{D_{12}}=\frac{\left|D_{12}^{\infty}-D_{12}^{\infty, r e f}\right|}{D_{12}^{\infty, r e f}} .
$$

The evolution of the relative error as a function of the mesh size is shown in Figure 3a. The coupled numerical procedure appears to converge as $(\Delta x / \ell)^{2}$. We note that the difference between $N_{E}=5120$ and $N_{E}=8192$ is less than $3 \times 10^{-4}$. This justifies using only 5120 elements in the results reported here (except when specified otherwise).

The temporal convergence is then verified using as reference $D_{12}^{\infty, \text { ref }}$ the value of $D_{12}^{\infty}$ obtained for $\dot{\gamma} \Delta t=1 \times 10^{-4}$ and $N_{E}=5120$. The evolution of the relative error as a function of the dimensionless time step is shown in Figure $3 \mathrm{~b}$. We find that the relative error remains small $\left(\epsilon_{D_{12}}<3 \times 10^{-3}\right)$ and that the numerical procedure converges linearly in time. 

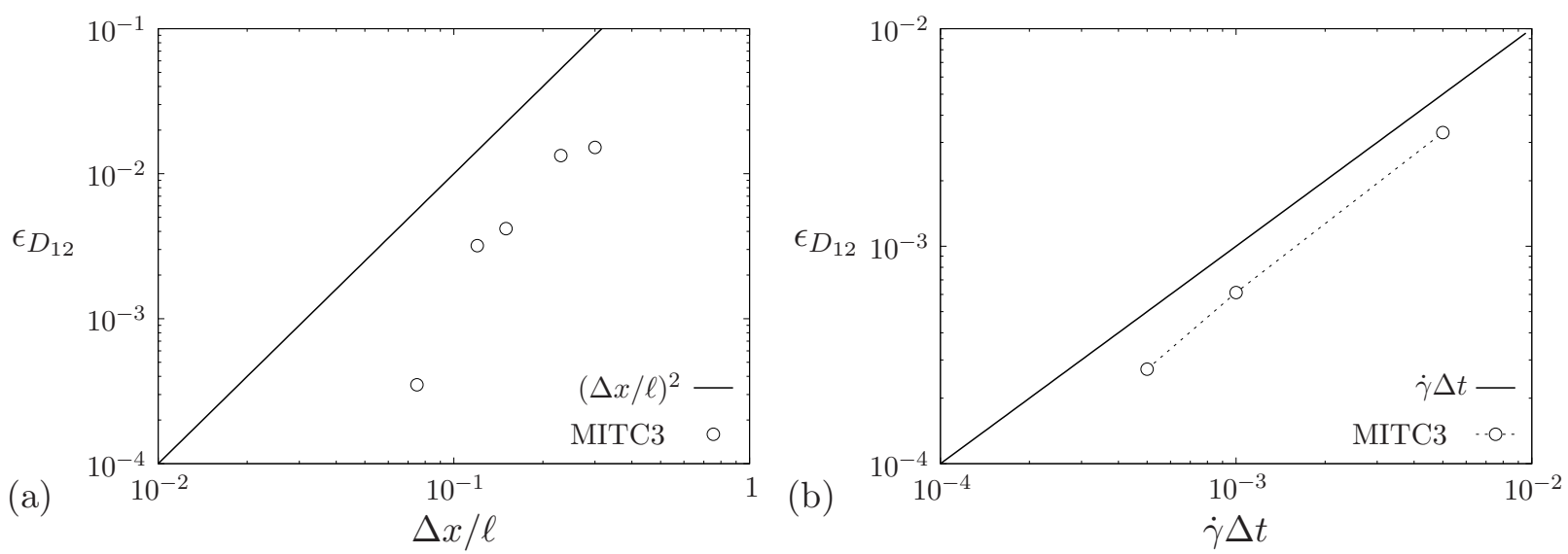

FIG. 3. Relative numerical error $\epsilon_{D_{12}}$ based on the Taylor parameter $D_{12}^{\infty}$ for $C a_{s}=0.6, \alpha=0.01$.

(a) Spatial convergence (reference corresponding to $\dot{\gamma} \Delta t=1 \times 10^{-4}, N_{E}=8192$ ); (b) Time convergence (reference corresponding to $\dot{\gamma} \Delta t=1 \times 10^{-4}, N_{E}=5120$ ).

\section{EFFECT OF WALL BENDING RESISTANCE ON THE DEFORMATION OF A CAPSULE}

We investigate the influence of two parameters on the dynamics of an initially spherical capsule subjected to a simple shear flow: the capillary number and the wall thickness (or equivalently the bending number). In the following, all the results (capsule profiles, deformation, etc.) pertain to the deformed capsule mid-surface.

\section{A. Deformation of a capsule with no bending resistance}

An initially spherical capsule with no bending resistance $(\alpha=0)$ subjected to a simple shear flow is elongated in the straining direction ${ }^{9-12}$. This is illustrated in Figure 4 for a capsule with a membrane satisfying the generalized Hooke's law. At low flow strength, compressive tensions (forces per unit curved length in the capsule surface) arise in the central region during the transient phase and persist at steady state. They lead to membrane buckling and to the formation of wrinkles with a half-wavelength that is a function of the grid spacing (Figure 4a). The wrinkles are purely numerical, as no wall bending resistance is contained in the model, but the location where they occur is physical: it correlates with regions where the membrane is undergoing compression. When the surface capillary number increases above the critical value $C a_{s L} \sim 0.4-0.45$ (irrespective of the wall constitutive law, because the deformation is still small), the capsule is more elongated: the tensions in the 


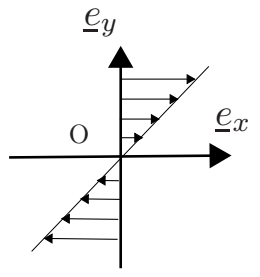

(a)

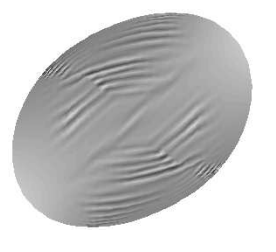

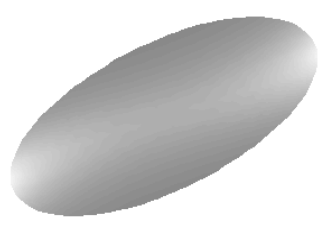

(b)

(c)

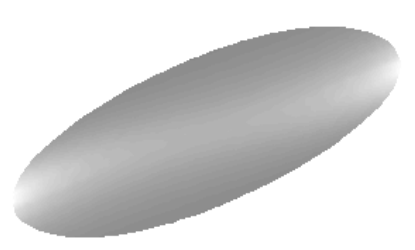

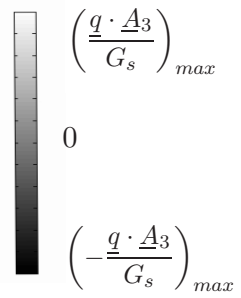

FIG. 4. Steady shape of an initially spherical capsule with $\alpha=0$ subjected to a simple shear flow $\left(N_{E}=8192\right):(a) C a_{s}=0.1 ;(b) C a_{s}=0.6 ;(c) C a_{s}=1.2$. The grey level represents the repartition of the normal load, where the maximum value $\left(\underline{q} \cdot \underline{A}_{3} / G_{s}\right)_{\max }$ is equal to $(a) 0.5,(b) 3$ and $(c) 4.5$.

membrane increase and become all positive, so that wrinkles no longer appear at steady state (Figures 4b-c).

\section{B. Effect of wall thickness}

In order to study the influence of the wall thickness on the capsule deformability, we simulate capsules made of the same homogeneous 3D material but with different wall thicknesses, subjected to a linear shear flow corresponding to $C a_{v}=0.05$. The profile of the capsule mid-surface in the shear plane is shown in Figure 5a. It shows that the thinner the wall, the more elongated the capsule becomes under the influence of the external flow. The capsule deformation decreases when the wall thickness increases. The tip curvature also decreases, so that the capsule has a more rounded shape. The steady-state deformation $D_{12}^{\infty}$ of the capsule mid-surface was computed from the corresponding ellipsoid of inertia. Figure 5b indicates that, for a given value of $C a_{v}$, i.e. for a given wall material, the deformation decreases as the thickness increases.

We now study the combined effects of $C a_{v}$ and $\alpha$. For a given wall thickness, the deformation increases when $C a_{v}$ increases, since the capsule is more elongated by the external flow (Figure 6a). When the wall thickness is increased, we find again that the deformation decreases. Increasing the wall thickness thus has the drastic effect of preventing the capsule from deforming. It is interesting, however, that, if one plots the deformation as a function of the surface capillary number $C a_{s}$, all the results fall onto one curve, which is the one obtained for a membrane wall devoid of bending resistance (Figure 6b). This means that 


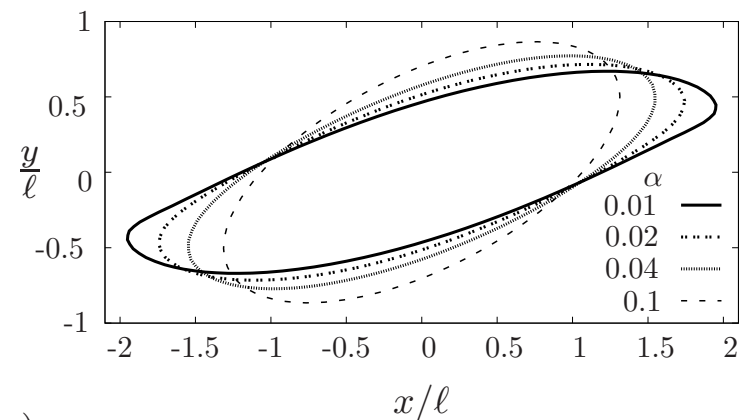

a)

FIG. 5. Capsule with wall material defined by $C a_{v}=0.05$ : influence of wall thickness $(a)$ on the mid-surface profile in the shear plane and $(b)$ on the mid-surface deformation at steady-state $D_{12}^{\infty}$.
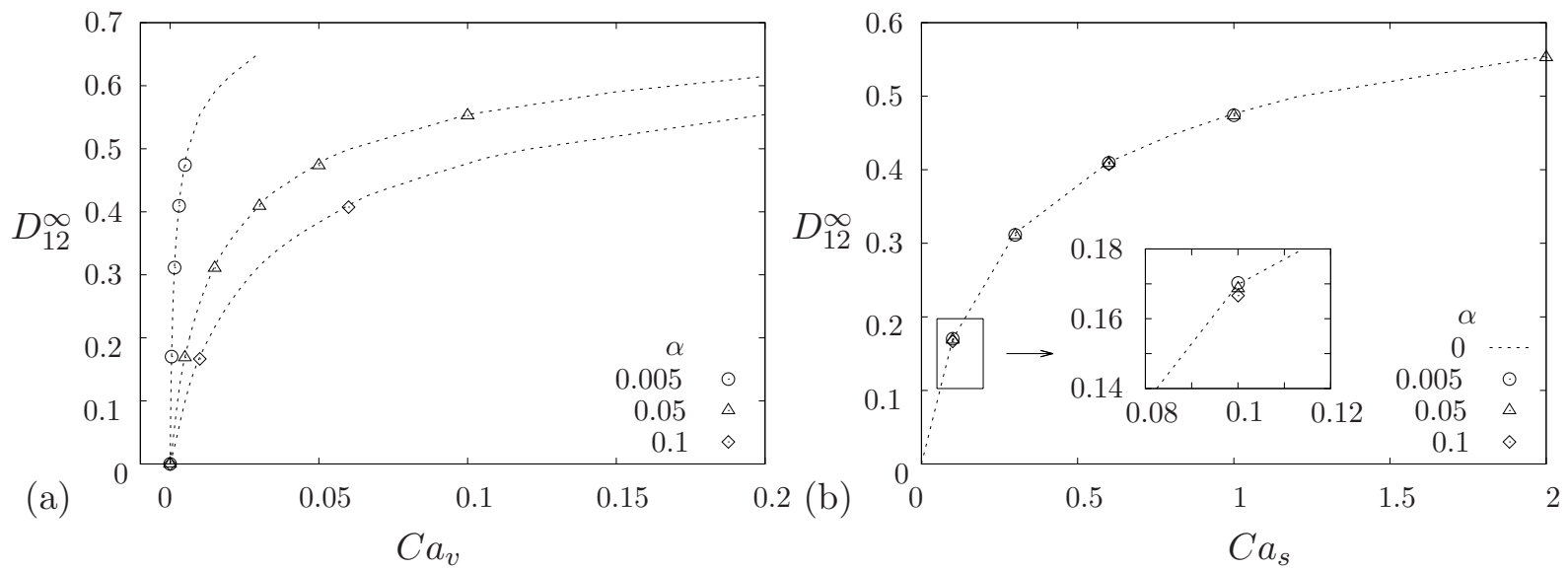

FIG. 6. Mid-surface deformation at steady state $D_{12}^{\infty}$ as a function of $(a) C a_{v}$ and of (b) $C a_{s}$ for different thicknesses.

even under large deformation, the bending resistance has a negligible effect on the overall capsule deformed profile and that the main mode of deformation results from the stretching of the mid-surface $\mathcal{S}_{t}$ in its plane (membrane mode).

\section{Membrane buckling at low flow strength}

We now study the effect of bending resistance (or equivalently of shell thickness) on the behavior of a capsule for a constant $C a_{s}$. We consider the case $C a_{s}=0.1$, which is below the critical value $C a_{s L}$, in order to be in the regime where buckling is known to occur for zero-thickness capsules. The wall thickness is varied between $\alpha=0.005$ and 0.02 . The corresponding bulk capillary number $C a_{v}$ is small and varies between $5 \times 10^{-4}$ and $2 \times 10^{-3}$. 

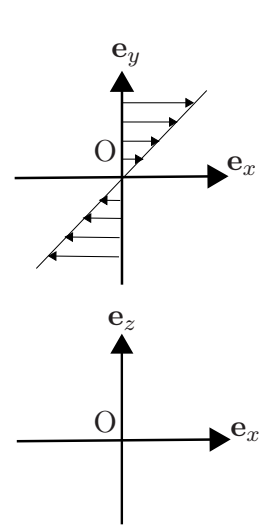

(a)

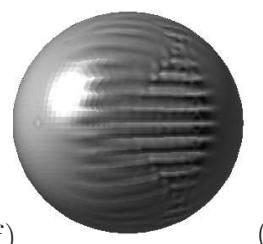

(b)

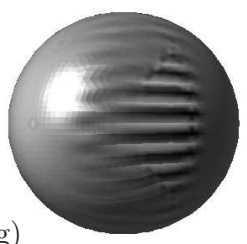

(c)

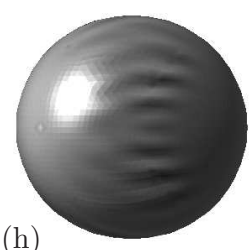

(d)
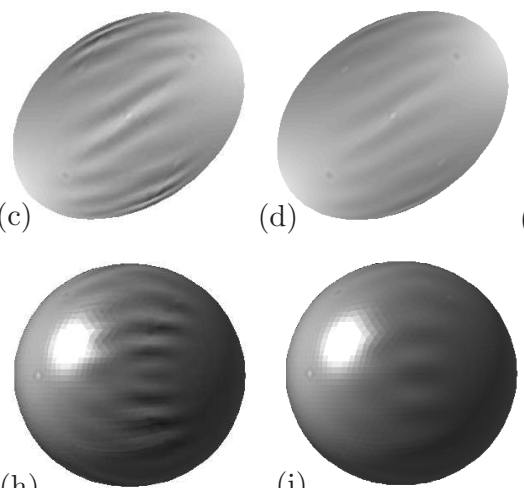

(i)

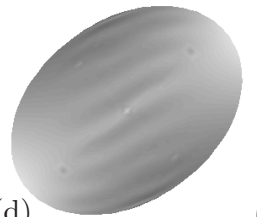

(e)

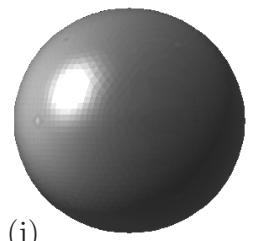

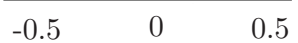

FIG. 7. Steady profiles of an initially spherical capsule subjected to a simple shear flow at $C a_{s}=0.1$ for different wall thicknesses $\alpha\left(N_{E}=8192\right)$. $(a)$ to $(e)$ : projection in the shear $x y$-plane; $(f)$ to $(j)$ : projection in the shear $x z$-plane. The grey levels represent the repartition of the normal load, the white spots on views $(f)$ to $(j)$ are a lighting artefact.

When the capsule wall is treated as a thin shell, it is still subjected to compressive tensions in the central region as it was observed in the membrane model. As a consequence, buckling may occur at steady state depending on the bending resistance of the capsule wall (Figure 7). For very small thicknesses (e.g. $\alpha=0.003$ ), the wrinkles that can be observed in Figure 7a appear at the same location and with the same pattern as those in Figure 4a, which corresponds to a capsule devoid of bending stiffness. When the wall thickness is increased, the bending resistance increases and fewer wrinkles are formed (Figure 7b-d). For $\alpha \geq 0.02$, the wall is too thick for wrinkles to form (Figures $7 \mathrm{e}, 7 \mathrm{j}$ ), even if it is subjected to compressive tensions. It is thus possible to prevent buckling by increasing the wall thickness (or equivalently the bending resistance).

\section{Wrinkle analysis}

It is not very easy to study the geometric characteristics of the wrinkles (amplitude, wavelength), as the capsule is a three-dimensional closed object. We first consider the same case as in the previous subsection of a capsule subjected to a surface capillary number $C a_{s}=0.1$. The capsule has a thickness ratio $\alpha=0.01$. The most reproducible technique 
(a)

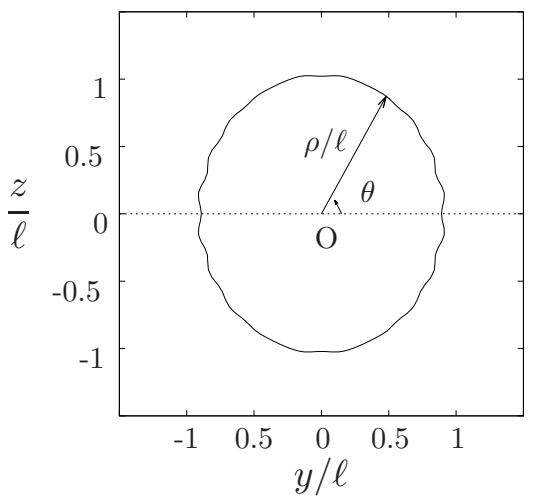

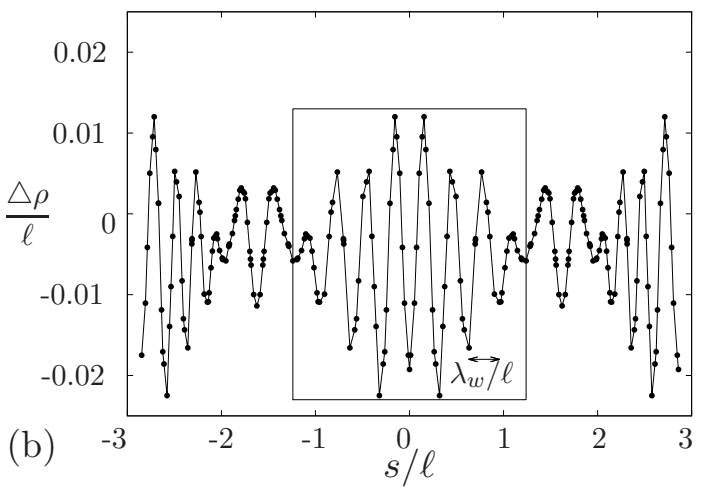

FIG. 8. Membrane wrinkles for $C a_{s}=0.1, \alpha=0.01, N_{E}=8192, \Delta x / \ell=0.06$. (a) Capsule midsurface profile in the $y z$-plane (the dotted line represents the shear plane); $(b)$ radius difference between the ellipsoid of inertia and the mid-surface in the $y z$-plane. The arc length is measured along the profile with $s=0$ when $\theta=0$, the points represent intersections with the grid.

is to study the wrinkles in the $y z$-plane. Their characteristics are found from the capsule cross-section $\mathcal{S}_{y z}$ within the plane: we subtract from the in-plane capsule profile the profile of the ellipsoid of inertia in the same plane. The capsule profile is shown in Figure 8a in terms of the polar coordinates $(\rho, \theta)$, such that the shear plane is located at $\theta / \pi=0,-1$. The radius difference $\Delta \rho / \ell$ between the two curves is shown in Figure $8 \mathrm{~b}$ as a function of the arc length $s / \ell$ along $\mathcal{S}_{y z}$, where $s=0$ when $\theta=0$.

One must note that the profile oscillations $\Delta \rho / \ell$ are determined from the profile intersection with the $y z$-plane, which cuts through the surface grid and intersects the elements randomly. It follows that the $\Delta \rho / \ell$ versus $s / \ell$ curve has an uneven spatial discretization with mean value $\Delta x / 2 \ell$. In particular for the case shown in Figure $8 \mathrm{~b}$, we have between 9 and 12 points per oscillation, which is enough to determine a wavelength.

To determine a characteristic wrinkle wavelength at a given time $\dot{\gamma} t$, we measure the distance between two successive extrema for the wrinkles located near the shear plane $(s / \ell \in$ $[-1.28,+1.28]$ - see the box in Figure $8 \mathrm{~b})$, which is the zone where the wrinkles are the most apparent, and compute the wrinkle mean wavelength $\lambda_{w} / \ell$. The wrinkle wavelength $\lambda_{e}$ in the equatorial plane of the capsule is then $\lambda_{w} \cos \Theta$, where $\Theta$ is the angle between the capsule long axis in the shear plane and the $x$-axis at steady state (Figure 2). A characteristic wrinkle amplitude $A / \ell$ is determined from the wrinkle located at $s=0$ (wrinkle with the maximum amplitude).

Prior to studying the wrinkle characteristics, we first need to find the mesh refinement for 


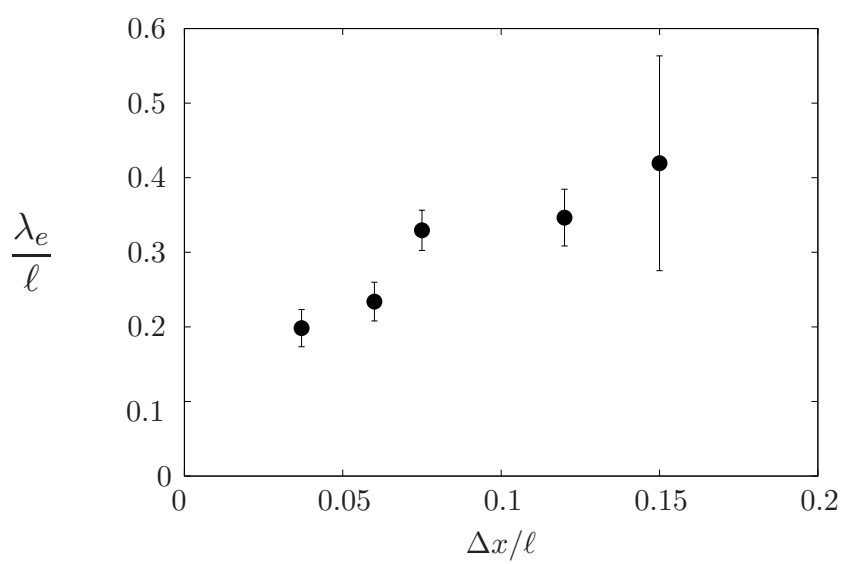

FIG. 9. Influence of the mesh size $\Delta x / \ell$ on the space- and time-average wrinkle wavelength $\left(C a_{s}=0.1, \alpha=0.01\right)$.

which the wrinkle wavelength no longer depends on the mesh size. To do so, we determine the various wavelengthes of the wrinkles in the region of interest (boxed region shown in Figure $8 \mathrm{~b})$ and repeat the procedure at four instants of time $(\dot{\gamma} t=3,3.5,4,4.5)$ to cover a quarter of the tank-treading period. Figure 9 shows the evolution of the space- and time-average value of the wrinkle wavelengthes as a function of the mesh size. The error bars correspond to the value of standard deviation, which is about $10 \%$ for $\Delta x / \ell \leq 0.12$ $\left(N_{E} \geq 2048\right)$ and $30 \%$ for $\Delta x / \ell=0.15\left(N_{E}=1280\right)$. We have added a very fine mesh $\left(N_{E}=20480, N_{N}=10242, \Delta x / \ell=0.037\right)$ for the sake of completeness. For a coarse mesh (e.g. $\Delta x / \ell=0.15, N_{E}=1280$ ), the rigidity due to the surface elements is dominant, so that the wrinkle wavelength $\left(\lambda_{e} / \ell \sim 0.36\right)$ is difficult to determine with great precision, but is approximately equal to twice the element spacing. When the number of elements increases, the mean wrinkle wavelength decreases and tends towards a limiting value $\lambda_{e} / \ell \sim 0.2$. Note that this plateau value becomes roughly independent of the mesh size when $\Delta x / \ell \leq 0.06$ $\left(N_{E} \geq 8192\right)$ and that it is much larger than the grid point spacing. The difference between the mean wavelength values computed with $\Delta x / \ell=0.06\left(N_{E}=8192\right)$ and $\Delta x / \ell=0.037$ $\left(N_{E}=20480\right)$ is less than $3.6 \times 10^{-2}$, which is of the same order of magnitude as the error. In the following, the results will therefore be obtained by considering a mesh with $N_{E}=8192$ elements. The most refined mesh $\left(N_{E}=20480\right)$ indeed requires several weeks of computation to reach the steady state. One can finally note that Figure 9 indicates that $\lambda_{e} / \ell \sim 0.2$ is the physical buckling wavelength of a shell of thickness ratio $\alpha=0.01$.

The influence of the thickness ratio on $\lambda_{e}$ is shown in Figure 10a at $\dot{\gamma} t=4.5$ for $C a_{s}=0.1$. 

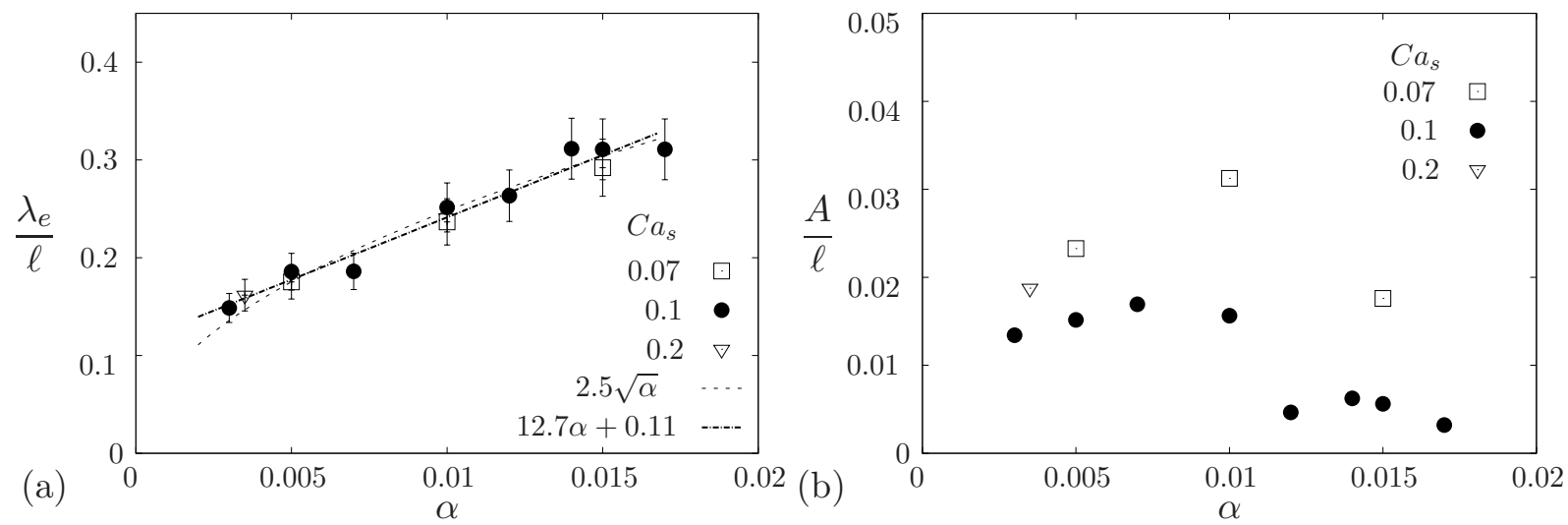

FIG. 10. Influence of the shell thickness on $(a)$ the wrinkle mean wavelength and $(b)$ amplitude for different values of $C a_{s}$.

We find that $\lambda_{e}$ increases with $\alpha$. If one increases the thickness (i.e. bending resistance), the wrinkles have a higher wavelength. Their amplitude remains about constant for low values of bending resistance (Figure 10b); it, however, strongly decreases hereafter when the bending resistance is increased, until being nil when the wrinkling phenomenon stops $\left(\alpha \geq 0.02\right.$ for $\left.C a_{s}=0.1\right)$.

In order to determine whether the surface capillary number $C a_{s}$ influences the wrinkle wavelength, we have also considered the cases $\left(C a_{s}=0.07, \alpha=0.005,0.01,0.015\right)$ and $\left(C a_{s}=0.2, \alpha=0.004\right)$. As shown in Figure 10a, the corresponding wrinkle wavelength follows the same trend for all the values of $C a_{s}$, and only depends on the relative wall thickness $\alpha$. This result is not unexpected, as the buckling behavior of the wall depends on its bending resistance. The wall thickness above which wrinkles no longer form, however, decreases when the surface capillary number $C a_{s}$ increases. The amplitude is indeed nil for $\alpha \geq 0.02$ at $C a_{s}=0.1$ (Figure 10b) and for $\alpha \geq 0.005$ at $C a_{s}=0.2$. This is due to the fact that, as $C a_{s}$ increases, the wall deformation increases and the amplitude of the compressive tensions decreases (recall that for a flow strength larger than $C a_{s L}$, there is no more compression and all the tensions are positive). Consequently, as $C a_{s}$ is increased, the compression on the wall decreases, so that less thickness is needed to withstand buckling. 


\section{DISCUSSION AND CONCLUSION}

The dynamics of an initially spherical capsule subjected to a linear shear flow has been studied numerically by coupling a shell finite element method with a boundary integral method to account for three-dimensional homogeneous wall properties and model the wall bending resistance. This method allows to study the influence of small thickness on the capsule deformation. It is numerically stable and free of locking.

We observe that, for a given wall material, the capsule deformability decreases when the wall thickness increases. However, if we consider only the dynamics of the shell mid-surface, we find that the overall deformation of the capsule depends only on the surface capillary number with no influence of the bending resistance. This means that the stretching of the mid-surface is the prevailing phenomenon. This is an important result, because it validates the use of a simple membrane model to compute the deformation of a capsule: such a model is indeed much faster and easier to run than a full shell model. Note that, if we use $C a_{s}$ as the main parameter for given flow strength and capsule size, the bulk elastic modulus $G$ implicitly decreases when $\alpha$ is increased. This means that the larger $\alpha$, the softer the material. This is probably the reason why we see no effect of thickness on the deformation curve in Figure 6b.

The sole dependence on $C a_{s}$ may also well be an effect of the facts that the capsule is initially spherical and that its core is entirely filled with an incompressible liquid. It can thus only deform by increasing its surface area, as its volume is constant. The capsule deformation thus results from elastic stretching of the wall. It follows that a simple membrane model is adequate to model the elastic behavior of spherical capsules, unless one is specifically interested in the post-buckling behavior. However, this may not be true for capsules which are initially non-spherical: such particles have an excess surface area compared to the enclosed volume and can change their geometry at small energy cost. In this case, bending effects may become preponderant compared to surface stretching.

Other authors such as Pozrikidis ${ }^{22}$, Le $^{23}$ and Hang et al. ${ }^{14}$ have observed that the bending resistance reduces the capsule deformability at a given $C a_{s}$. But they have all considered very large values of bending resistance that presently correspond to a wall thickness ratio $\alpha$ of 0.3 in the lowest case and up to 0.9: they, hence, do not respect the hypothesis of thin shell. It is difficult to imagine an existing homogeneous (or even composite) material that 
has both a very low shear elastic modulus and a very high bending modulus. The case of the red blood cell membrane is the exception, but its wall can hardly be considered as being homogeneous.

Even if a membrane model can be used to predict the post-buckling overall shape of a thin spherical capsule, a shell model is needed to analyze the formation of the wrinkles, which appear at low $C a_{s}$. We have found that the wrinkle wavelength depends only on the wall thickness ratio $\alpha$ (or equivalently the bending number $K_{b}$ ) and increases with it. The wavelength can be correlated to the bending stiffness either linearly:

$$
\lambda_{e} / \ell=12.7 \alpha+0.11
$$

or by a power function:

$$
\lambda_{e} / \ell=2.5 \sqrt{\alpha}=3.3\left(K_{b}\right)^{1 / 4}
$$

with a correlation coefficient $R^{2}=0.98$ in both cases. It is difficult to decide numerically between the two correlations, as it takes very long computational times to go below $\lambda_{e} / \ell=$ 0.2. Indeed, this requires to impose very small wall thickness $(\alpha \ell<0.003)$ and to use an extremely fine mesh with a correspondingly small time step (Eq. 19). However in principle, the wrinkle wavelength should decrease to the asymptotic value of zero for $\alpha=0$, which corresponds to a membrane with no resistance to bending. So it would seem that the correlation (Eq. 28) is physically more realistic than (Eq. 27). It is interesting to note that Cerda and Mahadevan ${ }^{24}$, who studied a thin homogeneous membrane stretched between two clamped ends, have predicted that the wavelength $\lambda_{e} / \ell$ of the wrinkles forming on the sheet is a function of the square root of the thickness ratio and have found the correlation law $\sqrt{\frac{8 \pi}{3}} \sqrt{\alpha}=2.9 \sqrt{\alpha}=3.8\left(K_{b}\right)^{1 / 4}$. The small difference between the factors of proportionality between Cerda and Mahadevan's correlation law and ours (Eq. 28) is due to the difference in geometry and boundary conditions. It seems to indicate that the wavelength of the wrinkles forming along the capsule can be predicted from a simple energy balance between bending and stretching under the constraint of the constitutive law of the wall material. However, if this balance is relatively simple to establish analytically for a flat membrane, it is much harder to find for a spherical membrane subjected to viscous shear forces. A numerical model is thus necessary in the latter case.

This result is essential from both a fundamental and an applied point of view. It can be used in practice to determine the capsule wall properties (thickness, surface shear modulus 

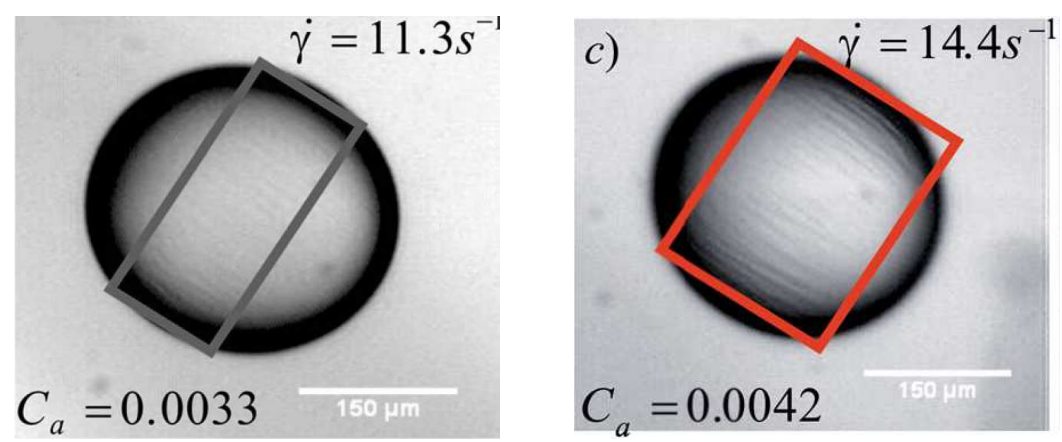

FIG. 11. Polysiloxane capsule subjected to a simple shear flow. Wrinkles (inside the rectangle) appear in the central region due to compressive tensions. The capillary number $C_{a}$ denoted on the pictures corresponds to the surface capillary number $C a_{s}$. Reproduced from I. Koleva and H. Rehage, "Deformation and orientation dynamics of polysiloxane microcapsules in linear shear flow", Soft Matter 8, 3681-3683 (2012, with permission of The Royal Society of Chemistry DOI: 10.1039/C2SM07182G).

and bending modulus) from experiments, in which an initially spherical capsule is subjected to a linear shear flow with $C a_{s}<C a_{s L}$. The idea is to first compute the Taylor parameter $D_{12}$ from an image of the deformed capsule shape acquired in the shear plane. The capillary number is deduced from the curve giving $D_{12}$ as a function of the surface capillary number (Figure 6b). Its value is independent of the material behavior, as the deformation is small at low flow strength. The surface shear modulus is then deduced from the definition of the surface capillary number $C a_{s}$ (Eq. 17) and the experimental values of $\dot{\gamma}$ and $\mu$. The second part of the procedure consists in measuring the wavelength of the wrinkles on the experimental image of the capsule. Knowing the wavelength $\lambda_{e}$, the wall thickness ratio can be estimated from Figure 10a or Eq. (28) and the bending modulus deduced from Eq. (13).

Using this method, we have analyzed the wrinkling processes of the polysiloxane capsules studied by Koleva \& Rehage ${ }^{4}$, which have a radius equal to $\ell=163 \mu \mathrm{m}$ (Figure 11 ). The capsule surface shear modulus is estimated from the small deformation asymptotic relation ${ }^{5}$

$$
D_{12}=\frac{25}{12} \frac{\mu \dot{\gamma} \ell}{G_{s}}
$$

and found to be equal to $2.19 \times 10^{-1} \mathrm{~N}^{-1} \mathrm{~m}^{-1}$. The wavelength of the folds can be measured to be roughly $10.4-12.9 \mu \mathrm{m}$. We find a value of wall thickness equal to $\alpha \ell \sim(107-163) \times$ $10^{-9} \mathrm{~m}$, and a bending modulus $M_{b} \approx 0.8-1.9 \times 10^{-3}$ N.m. The present analysis thus offers 
an interesting technique to characterize the mechanical properties of capsules of micrometric size and to provide an estimation of an apparent mean thickness of the wall.

In principle, the results of this paper are restricted to thin capsules. In the previous paragraph, we have just shown that the wall thickness of interface cross-linked capsules is about a thousand times smaller than the radius, which confirms the validity of the thinshell approximation to study such artificial capsules. If we want to model thicker capsules, one should probably switch to a three-dimensional model for the capsule wall, an endeavor which is outside the scope of this paper.

\section{ACKNOWLEDGEMENTS}

This research was funded by Ecole Polytechnique (PhD scholarship), the Conseil Régional de Picardie (MODCAP grant), by the French Ministère de la Recherche (Pilcam2 grant), by Sorbonne Université (B3Caps) and by the French Agence Nationale de la Recherche (CAPSHYDR grant ANR-11-BS09-013, Labex MS2T ANR-11-IDEX-0004-02).

\section{Appendix A: Non-linear expression of the Green-Lagrange strain tensor}

To find the expression of the non-linear Green-Lagrange strain tensor in the threedimensional contravariant base $\left(\underline{G}^{1}, \underline{G}^{2}, \underline{G}^{3}\right)$, it is convenient to introduce the three-dimensional covariant base $\left(\underline{G}_{1}, \underline{G}_{2}, \underline{G}_{3}\right)$ in the reference configuration defined by

$$
\underline{G}_{\alpha}=\underline{X}_{, \alpha}=\underline{A}_{\alpha}+\xi^{3} \underline{A}_{3, \alpha} \quad \text { and } \quad \underline{G}_{3}=\underline{A}_{3} .
$$

The components of the three-dimensional metric tensor $G_{i j}$ are

$$
\left\{\begin{array}{l}
G_{\alpha \beta}=\underline{G}_{\alpha} \cdot \underline{G}_{\beta}, \\
G_{\alpha 3}=\underline{G}_{\alpha} \cdot \underline{G}_{3}=0, \\
G_{33}=\underline{G}_{3} \cdot \underline{G}_{3}=1
\end{array}\right.
$$

and the components of the three-dimensional contravariant base $\left(\underline{G}^{1}, \underline{G}^{2}, \underline{G}^{3}\right)$ are found as previously using $\underline{G}^{\alpha} \cdot \underline{G}_{\beta}=\delta_{\beta}^{\alpha}$.

In the three-dimensional contravariant base, the definition of the Green-Lagrange strain 
tensor becomes

$$
\begin{aligned}
\underline{e} & =\frac{1}{2}\left(\underline{F}^{T} \cdot \underline{F}-\underline{G}\right), \\
& =\frac{1}{2}\left(g_{i j}-G_{i j}\right) \underline{G}^{i} \otimes \underline{G}^{j}, \\
& =\frac{1}{2}\left(\underline{u}_{, i} \cdot \underline{G}_{j}+\underline{u}_{, j} \cdot \underline{G}_{i}+\underline{u}_{, i} \cdot \underline{u}_{, j}\right) \underline{G}^{i} \otimes \underline{G}^{j},
\end{aligned}
$$

where $g_{i j}$ is the three-dimensional metric tensor in the current configuration defined by $g_{i j}=\underline{g}_{i} \cdot \underline{g}_{j}$ with the covariant base vector given by

$$
g_{i}=\underline{x}_{, i}
$$

in the deformed configuration. Using the definitions of the displacement field (Eq. 6) and of the covariant base vectors (Eq. A1, A3), the non-linear Green-Lagrange strain tensor (Eq. A2) can be written under the Reissner-Mindlin kinematical assumption as

$$
\left\{\begin{array}{l}
e_{\alpha \beta}=\gamma_{\alpha \beta}\left(\underline{u}_{s}\right)+\xi^{3} \chi_{\alpha \beta}\left(\underline{u}_{s}, \underline{\theta}\right), \\
e_{\alpha 3}=\zeta_{\alpha}\left(\underline{u}_{s}, \underline{\theta}\right), \\
e_{33}=0 .
\end{array}\right.
$$

The tensors $\underline{\gamma}$ and $\underline{\underline{\chi}}$ are the membrane strain and bending strain tensors respectively and the vector $\underline{\zeta}$ the shear strain vector. In $(\mathrm{A} 4)$, the components of $e_{\alpha 3}$ depending on $\xi^{3}$ have been neglected (since the zero-order term can itself be proved to be of the order of the thicknesses at solution), as well as the components of $e_{\alpha \beta}$ depending on $\left(\xi^{3}\right)^{2}$. The expressions of $\underline{\underline{\gamma}}, \underline{\underline{\chi}}$ and $\underline{\zeta}$ are respectively:

$$
\begin{aligned}
\gamma_{\alpha \beta} & =\frac{1}{2}\left[\underline{A}_{\alpha} \cdot \underline{u}_{s, \beta}+\underline{A}_{\beta} \cdot \underline{u}_{s, \alpha}+\underline{u}_{s, \alpha} \cdot \underline{u}_{s, \beta}\right], \\
\chi_{\alpha \beta} & =\frac{1}{2}\left[\underline{u}_{s, \alpha} \cdot \underline{A}_{3, \beta}+\underline{u}_{s, \beta} \cdot \underline{A}_{3, \alpha}+\underline{\theta}_{, \alpha} \cdot \underline{A}_{\beta}+\underline{\theta}_{, \beta} \cdot \underline{A}_{\alpha}+\underline{\theta}_{, \beta} \cdot \underline{u}_{s, \alpha}+\underline{\theta}_{, \alpha} \cdot \underline{u}_{s, \beta}\right], \\
\zeta_{\alpha} & =\frac{1}{2}\left[\underline{A}_{\alpha} \cdot \underline{\theta}+\underline{A}_{3} \cdot \underline{u}_{s, \alpha}+\underline{\theta} \cdot \underline{u}_{s, \alpha}\right] .
\end{aligned}
$$

\section{REFERENCES}

${ }^{1}$ F. Edwards-Lévy, M.-C. Andry, and M.-C. Lévy, "Determination of free amino group content of serum albumin microcapsules: II. Effect of variations in reaction time and in terephthaloyl chloride concentration," Int. J. Pharm. 103, 253 - 257 (1994). 
${ }^{2}$ Z. Y. Xiang, Y. C. Lu, Y. Zou, X. C. Gong, and G. S. Luo, "Preparation of microcapsules containing ionic liquids with a new solvent extraction system." React. Funct. Polym. 68 (8), $1260-1265$ (2008).

${ }^{3}$ T. X. Chu, A.-V. Salsac, E. Leclerc, D. Barthès-Biesel, H. Wurtz, and F. Edwards-Lévy, "Comparison between measurements of elasticity and free amino group content of ovalbumin microcapsule membranes: Discrimination of the cross-linking degree," J. Colloid Interf. Sci. 355, $81-88$ (2011).

${ }^{4}$ I. Koleva and H. Rehage, "Deformation and orientation dynamics of polysiloxane microcapsules in linear shear flow." Soft Matter 8, 3681 - 3693 (2012).

${ }^{5}$ D. Barthès-Biesel, "Modeling the motion of capsules in flow," Curr. Opin. Colloid In. 16, $3-12(2011)$.

${ }^{6}$ K. S. Chang and W. L. Olbricht, "Experimental studies of the deformation and breakup of a synthetic capsule in steady and unsteady simple shear flow," J. Fluid Mech. 250, 609 - 633 (1993).

${ }^{7}$ A. Walter, H. Rehage, and H. Leonhard, "Shear-induced deformation of polyamid microcapsules," Colloid Polymer Sci. 278, 169 - 175 (2000).

${ }^{8}$ A. Walter, H. Rehage, and H. Leonhard, "Shear induced deformation of microcapsules: shape oscillations and membrane folding," Colloid Surface A 183 - 185, 123 - 132 (2001).

${ }^{9} \mathrm{~S}$. Ramanujan and C. Pozrikidis, "Deformation of liquid capsules enclosed by elastic membranes in simple shear flow: Large deformations and the effect of capsule viscosity," J. Fluid Mech. 361, 117 - 143 (1998).

${ }^{10}$ E. Lac, D. Barthès-Biesel, A. Pelakasis, and J. Tsamopoulos, "Spherical capsules in threedimensional unbounded Stokes flows: effect of the membrane constitutive law and onset of buckling," J. Fluid Mech. 516, 303 - 334 (2004).

${ }^{11} \mathrm{X}$. Li and K. Sarkar, "Front tracking simulation of deformation and buckling instability of a liquid capsule enclosed by an elastic membrane," J. Comput. Phys. 227, 4998-5018 (2008).

${ }^{12}$ J. Walter, A.-V. Salsac, D. Barthès-Biesel, and P. Le Tallec, "Coupling of finite element and boundary integral methods for a capsule in a Stokes flow," Int. J. Num. Meth. Engng 83, 829 - 850 (2010).

${ }^{13}$ E. Foessel, J. Walter, A.-V. Salsac, and D. Barthès-Biesel, "Influence of internal viscosity on the large deformation and buckling of a spherical capsule in a simple shear flow," J. 
Fluid Mech. 672, 477 - 486 (2011).

${ }^{14}$ W.-X. Hang, C. B. Chang, and H. J. Sung, "Three-dimensional simulation of elastic capsules in shear flow by the penalty immersed boundary method." J. Comput. Phys. 231, 3340 - 3364 (2012).

${ }^{15}$ S. Kessler, R. Finken, and U. Seifert, "Swinging and tumbling of elastic capsules in shear flow," J. Fluid Mech. 605, 207 - 226 (2008).

${ }^{16}$ D. V. Le and Z. Tan, "Large deformation of liquid capsules enclosed by thin shells immersed in the fluid." J. Comput. Phys. 229, 4097 - 4116 (2010).

${ }^{17}$ D. Chapelle and K. J. Bathe, The Finite Element Analysis of Shells - Fundamentals (Computational Fluid and Solid Mechanics). Second Edition, edited by Springer (2011).

${ }^{18}$ J. Walter, A.-V. Salsac, and D. Barthès-Biesel, "Ellipsoidal capsules in simple shear flow: prolate versus oblate initial shapes," J. Fluid Mech. 676, 318 - 347 (2011).

${ }^{19}$ The library is registered at the Agence pour la Protection des Programmes under ref: IDDN.FR.001.030018.000.S.P.2010.000.20600. The base of the program is open source and available online: www-rocq.inria.fr/modulef.

${ }^{20}$ P.-S. Lee and K.-J. Bathe, "Development of MITC isotropic triangular shell finite elements," Comput. Struct. 82, 945 - 962 (2004).

${ }^{21}$ J.-L. Batoz and G. Dhatt, Modélisation des structures par éléments finis: Coques, edited by Hermès (1992).

${ }^{22} \mathrm{C}$. Pozrikidis, "Effect of membrane bending stiffness on the deformation of capsules in simple shear flow," J. Fluid Mech. 440, 269 - 291 (2001).

${ }^{23}$ D. V. Le, "Effect of bending stiffness on the deformation of liquid capsule enclosed by thin shells in shear flow." Phys. Rev. E 82, 016318 (2010).

${ }^{24}$ E. Cerda and L. Mahadevan, "Geometry and physics of wrinkling," Phys. Rev. Lett. 90, $074302(2003)$. 